\title{
La investigación universitaria en periodismo científico
}

\author{
Dra. Carolina Moreno Castro \\ Profesora Titular de Periodismo Especializado de la Universidad de Valencia
}

\section{RESUMEN}

En este artículo, la autora revisa y examina las aportaciones bibliográficas sobre comunicación científica que se han realizado desde distintas áreas de conocimiento, en las últimas décadas en nuestro país. Así pues, muestra las debilidades presentes en los escasos estudios sobre el tratamiento informativo de la ciencia y la tecnología, realizados desde las facultades de Ciencias de la Información y la fortaleza de los trabajos abordados desde las facultades de Ciencias Experimentales donde hay abierta una línea de investigación sobre historia del periodismo científico.

\section{ABSTRACT}

In this article, the author revises and she examines the bibliographical contributions about scientific communication that have been carried out from different knowledge's areas, in the last decades in our country. Therefore, she shows the debilitys in the limited studies about informative treatment of the science and the technology, carried out from the facultys of communication sciences and the strengths of the works approached from the facultys of experimental sciences where they are opening an investigation line about history of the scientific journalism.

Palabras claves: Comunicación científica/Periodismo científico/Divulgación científica/Historias especializadas.

Key words: Scientific communication/Scientific journalism/Scientific popularization/Specialized histories.

\section{Periodismo científico versus revistas especializadas}

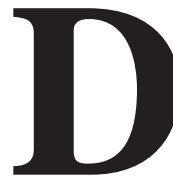
esde que se iniciaron en los años setenta los primeros estudios empíricos sobre periodismo científico se ha conformado un corpus teórico en el que se han incluido aleatoriamente trabajos sobre el tratamiento informativo y la divulgación científica en los medios de comunicación de información general y sobre revistas y boletines científicos especializados. En este artículo partimos de que el periodismo científico y la prensa científica se diferencian conceptual y epistemológicamente. El periodismo científico se concibe 
desde la proyección profesional de quienes trabajan en esta especialización de los flujos mediáticos en los diarios de información general o en cualquier otro medio de comunicación social. Asimismo, la prensa científica está dirigida por especialistas o expertos de las distintas disciplinas del saber y, por tanto, su difusión es restringida a grupos cualificados. Nos referimos a las revistas científicas, los boletines de las asociaciones profesionales, los boletines de los colegios profesionales y los boletines de las academias de las ciencias, entre otros, que son publicaciones especializadas y que no estarían circunscritas a nuestro ámbito de investigación. Sin embargo, comprobamos que desde la investigación universitaria existen interesantes aportaciones historiográficas llevadas a cabo desde áreas de conocimiento diferentes del periodismo que, sin duda, resultan imprescindibles para contextualizar esta propuesta de investigación.

Una vez revisada la bibliografía publicada en todo el Estado sobre periodismo científico, se puede afirmar taxativamente que hay una gran laguna sobre estudios empíricos, abordados desde las facultades de Ciencias de la Comunicación, en esta especialización periodística. Así las cosas, los primeros trabajos que se desarrollaron, durante los años setenta, en torno al periodismo científico procedían del ámbito profesional, siendo Manuel Calvo Hernando el pionero en estos primeros trabajos sobre periodismo y divulgación científica ${ }^{1}$. Parece lógico inferir que el desfase intelectual de esta especialización periodística es un reflejo de la falta de sensibilidad registrada en los planes de estudios de las facultades de Ciencias de la Comunicación. Hoy día, la situación ha cambiado razonablemente pues en la mayor parte de los planes de estudios de las facultades se contemplan en los segundos ciclos asignaturas optativas que tienen que ver con cualquier ámbito de la comunicación científica.

En este sentido, la Universidad de Valencia ha optado por la incorporación en la Licenciatura de Periodismo de un itinerario sobre 'ciencia y tecnología'. Las futuras líneas de investigación que surjan a partir de este escenario de formación universitaria todavía no han sido fecundas, por su inminente implantación. Como consecuencia de esta realidad, la investigación en comunicación científica ha avanzado muy lentamente, en el periodo cronológico que abarca desde los años setenta hasta la actualidad. Ésta es una de las razones por las que las investigaciones realizadas han estado, fundamentalmente, encaminadas al estudio de secciones especializadas o tematizadas de los periódicos de información general. No existe un planteamiento teórico que contemple una visión de conjunto de su contexto económico, político y social, desde una Historia del Periodismo Científico o ni si

1 Nos referimos a las primeras publicaciones que este autor abordó a finales de los setenta y cuya línea de investigación ha continuado durante las tres últimas décadas. Entre otras, vid. CALVO HERNANDO, Manuel: Civilización tecnológica e información, Mitre, Barcelona, 1982; Ciencia y Periodismo, CEFI, Barcelona, 1990, y Manual de Periodismo científico, Paraninfo, Madrid, 1977, 1ª edición, 2a 1992. 
quiera desde una visión más globalizadora sobre una Historia de la Comunicación Científica.

A grandes rasgos, las historias nacionales del periodismo contemporáneo han dedicado menor atención a las publicaciones periódicas no diarias y especializadas que a los diarios de información general, al ser considerados medios rectores de la evolución periodística. Sin embargo, no hemos de olvidar que en los últimos años esta tendencia se ha completado, por no decir que se ha invertido, con la creciente proliferación de estudios de conjunto de carácter local y regional (repertorios, catálogos, estudios monográficos, etc.) que, con el propósito de conocer y profundizar en el patrimonio hemerográfico propio, han vuelto también la mirada hacia las publicaciones no diarias y especializadas ${ }^{2}$.

Según el profesor Espinet, quienes tratan las publicaciones especializadas suelen ser los expertos en los contenidos de los que se ocupa la revista, excepto en el caso de las publicaciones no periódicas de información general. Para este autor «los estudiosos con los que se habrá de entender quien pretenda indagar en este terreno se compondrá de críticos de cine, pedagogos, feministas, sociólogos, politólogos, literatos, historiadores del teatro, médicos, etc., sin olvidar a algún que otro periodista e incluso comunicólogo» ${ }^{3}$. Esta prensa constituye, según Espinet, un segundo frente informativo diferenciado y específico.

En 1975, Gèrard Chastagnaret apelaba a la necesidad de que los estudios de prensa tuvieran como objeto las revistas científicas, y aducía para ello razones cuantitativas y cualitativas. 4 "En general -escribía Chastagnaret- los estudios de prensa no acostumbran a tener como objeto las revistas científicas o profesionales. Se trata, sin embargo, de un sector en el seno de la producción periódica que merece una consideración que no debe medirse únicamente por su importancia cuantitativa, sino por la importancia de las informaciones que puede proporcionar sobre las condiciones de producción y de circulación de una parte determinada de la prensa, así como sobre la evolución del grupo profesional y del campo de la actividad en cuestión".

Transcurrido un cuarto de siglo, comprobamos que, en nuestro país, existen cuantiosas aportaciones historiográficas sobre revistas especializadas en ciencia y tecnología realizadas mayoritariamente desde áreas de conocimiento distintas a

2 Pese al impulso adquirido a partir de los años setenta, esta línea de investigación hemerográfica no es nueva en nuestro país, más bien al contrario viene a recuperar de un modo científico una tradición investigadora anterior, marcada, sobre todo, por la erudición.

3 ESPINET, F.: «El segundo frente informativo: Revistas y prensa especializada», en ÁLVAREZ, Jesús Timoteo et al. : Historia de los medios de comunicación en España. Periodismo, imagen y publicidad (1900-1990). Ariel Comunicación, Madrid, 1989, p. 50.

4 Cfr. CHASTAGNARET, Gèrard: "Un ejemplo de revista científica: la Revista Minera desde 1850 a 1914", en TUÑón DE LARA, Manuel et al:: Prensa y sociedad en España, 1820-1936. Edicusa, Madrid, 1975, p. 223. 
Periodismo. Se han acometido trabajos cuyos resultados de investigación suponen una aportación para una disciplina específica y, por tanto, están alejados inicialmente de la Historia del Periodismo. Estos estudios suelen ser descriptivos (se localizan los fondos, se clasifican y se estudian los parámetros de la publicación) y de contenido (se analizan los temas y se sistematizan), alejándose, normalmente, de los procesos sociales en los que se desarrollan este tipo de revistas. Para muchos autores, estos trabajos, que continuaremos examinando en las páginas siguientes, suponen una aproximación a los periodismos especializados en ciencia y tecnología.

\section{Aportaciones bibliográficas sobre la prensa científica y sobre el periodismo científico desde dos áreas de conocimiento: Historia de la Ciencia y Periodismo}

Es destacable el hecho de que numerosas revistas científicas hayan sido estudiadas desde el ámbito de la Historia de la Ciencia. Desde esta área de conocimiento se han realizado valiosas aportaciones en los últimos años a las distintas ramas especializadas de la ciencia y la tecnología. Cabría resaltar que todos estos trabajos están vinculados al estudio de la prensa científica; esto es, revistas de expertos 5 .

En un sentido heurístico, nos referimos a los estudios analíticos y descriptivos que versan sobre un corpus especializado de un área de conocimiento. Por ejemplo, el artículo de Antonio García del Moral; Carmen Ruíz García, y José R. Santiburcio López, del departamento de Historia de la Medicina de la Universidad de Córdoba, titulado "El periodismo sanitario en Córdoba desde sus comienzos

5 Para conocer el trabajo realizado por los expertos de las diferentes áreas del conocimiento científico, vid. las obras de los siguientes autores: CALANDRIA AMIGUETI, José María y CABRERA ALONSO, Juan Rafael: «Aportaciones de los oftalmólogos hispanoamericanos a La Crónica Oftalmológica (Cádiz, 18711882), en Actas de las II Jornadas de Historia de la Medicina Hispanoamericana. Universidad de Cádiz, Cádiz, 1989; CAMACHO IGLESIAS, Avelina: "Noticias pediátricas en el Observatorio Habanero (18441847)", en Op. cit., Universidad de Cádiz, Cádiz, 1989, pp. 185-191; DOÑA NIEVES, Francisco y CABRERA ALONSO, Juan Rafael: "La prensa médica hispanoamericana en la Revista Ibero-americana de Ciencias Médicas (1899-1916), en Op. cit., 1989, pp. 199-207; FERNÁNDEZ DUEÑAS, Ángel: "Una revista cordobesa del siglo XIX: La Andalucía Médica", en Boletín de la Real Academia de Córdoba de Ciencias, Bellas Letras y Nobles Artes, $\mathrm{n}^{\circ}$ 100, volumen II, 1979; LASARTE CALDERAY, José y HERRERA RODRÍGUEZ, Francisco: "Noticias hispanoamericanas en la prensa andaluza de los auxiliares sanitarios (1936-1936)", en Anales de la Real Academia de Medicina y Cirugía de Cádiz, 1, 1992. LÓPEZ FERNÁNDEZ, Carlos: "Análisis temático de la producción física recogida en los Anales de la Real Sociedad Española de Física y Química durante el período (1940-1975)", en Llull. Boletín de la Sociedad Española de Historia de las Ciencias, ${ }^{\circ}$ 9, 1986, pp. 105-126; FRONTERA IZQUIERDO, Pedro: "Análisis estadístico de la producción sobre raquitismo del periodismo español hasta 1914", en Asclepio, Revista de Historia de la Medicina y de la Ciencia, $\mathrm{n}^{\circ}$ 28, 1976, pp. 255-274; PINTO MOLINA, María: Vida de una publicación científica: El correo Erudito. Universidad de Granada, Granada, 1986; RAMOS, $\mathrm{M}^{\mathrm{a}}$ Dolores; CASTELLANOS, Jesús y CARRILLO, Juan L.: "Regeneracionismo, regionalismo y ciencia en la Málaga de comienzos de siglo: la revista Andalucía Científica (1903-1904), en Dynamis. Acta Hispanica ad Medicinae Scientarumque Historiam Illustrandam, vol. 5-6, 1986. SÁEZ VILLAVERDE, Raquel: "Los comienzos del periodismo médico español. Boletín de Medicina, Cirugía y Farmacia", en Boletín de la Real Sociedad Bascongada de Amigos del País, nº 54, tomo II, 1998, pp. 509-519. 
hasta 1970". En este artículo, publicado en la revista Intus, se realizó una aproximación a la prensa sanitaria en Córdoba, que ha permitido localizar 19 revistas aparecidas entre 1876 y 1964, incluyendo publicaciones sobre Medicina, Veterinaria, Farmacia, Practicantes y Divulgación 6 .

Estos estudios son muy interesantes para que los especialistas de las distintas disciplinas científicas conozcan, a través del análisis de las publicaciones, las técnicas empleadas en su área de investigación por los expertos, la evolución de esas técnicas a lo largo de las décadas, las principales tendencias en las líneas de investigación que se han abordado y la implantación social que se ha llevado a cabo de esa tecnología. Estos estudios, como fuentes hemerográficas, están contribuyendo a «construir» las distintas historias de cada una de las Ciencias.

Así pues en la revisión que hemos llevado a cabo de los trabajos que analizan el tratamiento de la información científica y tecnológica, publicados durante los años ochenta y noventa del siglo $\mathrm{XX}$, se han realizado investigaciones, fundamentalmente, desde dos áreas de conocimiento: a) desde Periodismo y b) desde Historia de la Ciencia.

\subsection{La investigación en comunicación científica desde Periodismo}

Por una parte, hemos localizado trabajos procedentes de las Facultades de Ciencias de la Información o de la Comunicación, publicados, principalmente, desde el área Periodismo, que se han especializado en el tratamiento que los descubrimientos científicos y que los avances tecnológicos han recibido en los medios de comunicación y, muy especialmente, en la prensa diaria ${ }^{7}$. Ésta es la línea de investigación que mayor atención recibe por parte de los investigadores, observándose, sin embargo, diferentes matices y enfoques en cada uno de ellos. Desde esta posición, podemos distinguir dos tipos de investigaciones: a) aquéllas que están basadas en el tratamiento informativo de hechos científicos y tecnológicos; y b) aquéllas que están basadas en la divulgación del conocimiento científico y técnico.

\section{a) Investigaciones sobre el tratamiento informativo de la ciencia}

En 1992 se defienden las dos primeras tesis doctorales sobre periodismo científico en la Universidad Complutense de Madrid. En la primera de ellas,

6 Vid. GARCÍA DEL MORAL, Antonio; RUÍZ GARCÍA, Carmen, y SANTIBURCIO LÓPEZ, José Ramón: "El periodismo sanitario en Córdoba desde sus comienzos hasta 1970", en Intus. Revista de las Unidades Docentes de Psicología Médica, nº 2, 1990, pp. 212-225.

7 Hay que considerar, en este sentido, la atención de los investigadores hacia la prensa diaria (especialmente la prensa de calidad o de elite), al considerarla el principal soporte de sus estudios, frente a otros medios de comunicación, probablemente de mayor incidencia social (por ejemplo, la televisión). No obstante, como se señala en el estudio de Durán Escriba -de quien hablamos más adelante-, una línea de investigación podría tener que ver con la influencia que la televisión tiene sobre el tratamiento informativo de la prensa. 
Tratamiento de la información científica en la prensa diaria española de María Alcalá Santaella Oria de Rueda, la investigadora realiza un análisis de contenido sobre la información publicada sobre el SIDA en los diarios de mayor difusión de todo el Estado. En la segunda de ellas, Análisis estructural de la información especializada en contenidos agroalimentarios de José Luis Murcia García Consuegra, el autor analiza los contenidos agroalimentarios en la prensa española, y compara los datos obtenidos con diarios y revistas especializadas del resto de los países de la UE. Esta tesis refleja el paso que se ha dado desde el periodismo agrario de escasa calidad, al periodismo agroalimentario, mucho más científico y con un perfil fundamentalmente económico. Dos años más tarde, en 1994, también en la Universidad Complutense de Madrid, Carlos Cachán Alcolea defiende la tesis doctoral Ideología subyacente de El País, El Mundo, La Vanguardia y El Correo Español a la luz de la información ecológica, en la que intenta demostrar en qué medida estos cuatro diarios de referencia aceptan teorías científicas, sin calibrar racionalmente los límites de su aplicación. Para el tratamiento informativo de un tema como la ecología, con matices ideológicos claramente definidos, el autor comprueba si la ideología manifiesta de estos diarios coincide con el posicionamiento ante temas de esta naturaleza.

En 1995, Santiago Ramentol i Massana defiende la primera tesis doctoral sobre contenidos científicos en la Universidad Autónoma de Barcelona. Este trabajo se titula: La gàbia de vidre: (anàlisi de la comunicació des de la ciència i proposta d'unes pautes de relació amb la societat). Al años siguiente, en 1996, también en la Universidad Autónoma de Barcelona, Xavier Durán Escriba defiende la tesis titulada: Tratamiento periodístico de dos hechos tecnológicos: los primeros Sputnik (1957) y la llegada a la luna (1969) en la prensa diaria de Barcelona. Este trabajo analiza cómo la prensa diaria de Barcelona trató el aspecto tecnológico, el sociopolítico y el periodístico en estos dos acontecimientos. Después de exponer las características, la historia y las funciones del periodismo científico, la interacción entre ciencia e ideología, la situación sociopolítica en cada época y un panorama histórico de la astronáutica, analiza cómo la prensa trató ambos hechos: el rigor científico, la visión -triunfalista o no- de la tecnología y, en el caso del viaje a la luna, la influencia de la televisión en el tratamiento periodístico. Para este autor, el tratamiento periodístico de la tecnología no es independiente, y así ha de ser analizado, en el contexto de la información especializada. Contexto que se extiende a las circunstancias sociales y políticas de la época en la que se desarrolla la actividad periodística. A partir del estudio de la información científica y tecnológica se puede conocer y estudiar una determinada etapa histórica. Otro aspecto a tener en cuenta en este trabajo es el estudio del tratamiento retrospectivo, a partir de la información generada en los diarios, de los aniversarios de los hechos que son objeto del análisis. 
Hasta el año 1999, no se defiende ninguna tesis relacionada con la información científica. Durante este año, Carmen del Puerto Varela defiende en la Universidad de La Laguna la tesis doctoral titulada Periodismo científico: la astronomía en titulares de prensa. Este estudio destaca la presencia creciente de la astronomía y especialidades afines en los medios. También en La Universidad de La Laguna, se defiende otra tesis sobre periodismo científico en 1999. Su autor, Antonio Alarco Hernández, titula la investigación Periodismo Científico en prensa diaria. Aspectos biomédicos. Un trabajo en el que se valora el espacio dedicado a los aspectos de biomedicina, a través de estudios hemerográficos, al tiempo que se realiza una valoración cualitativa de los contenidos desde el punto de vista del análisis de los géneros utilizados para la difusión del mensaje. Y al año siguiente, en 2000, también en la Universidad de La Laguna se defiende otra tesis doctoral sobre información científica Flujos de información entre científicos y prensa, de Carlos José Elías Pérez. Este trabajo analiza las disfunciones provocadas por los gabinetes de prensa de las instituciones científicas, especialmente del CSIC, así como los gabinetes de comunicación de las revistas Nature y Science. También en el año 2000, Manuel Calvo Hernando (pionero en los estudios sobre comunicación científica y autor de referencia para los estudiantes de Periodismo) defiende su tesis doctoral con el título La ciencia como material informativo, en la Universidad San Pablo/CEU de Madrid. En 2001, se defiende la última tesis doctoral sobre tratamiento informativo de la ciencia y lo hace quien suscribe este artículo con el título La biotecnología en la prensa diaria (1988-1998). Análisis y tendencias, utilizando como vector de la investigación, la biotecnología como fenómeno tecnocientífico y como acontecimiento de actualidad. Entre las conclusiones más destacables de esta investigación, se pueden citar las disfunciones observadas en los textos analizados como la espectacularización que la prensa hace de los nuevos descubrimientos, la presentación de forma abusiva de una técnica en otras aplicaciones regresivas; y, por lo general, hacer prospectiva de forma especulativa.

\section{b) Investigaciones sobre divulgación científica}

También hemos examinado las tesis doctorales que han tratado el ámbito de la divulgación científica hasta la actualidad. En 1990, en la Universidad Autónoma de Barcelona, Anna María Carrascal Triola defiende la tesis Analisi Semiotica d' imatges. El cas de les imatges de la divulgacio cientifica. En esta investigación se propone un punto de vista como concepto sintetizador capaz de agrupar los elementos caracterizadores de un tipo de discurso y el de un tipo de imágenes, vinculadas con la divulgación científica. En la Universidad Complutense, en 1995, Carlos Horacio Lozano Ascencio presenta la tesis La expresión/representación de catástrofes a través de su divulgación científica en los medios de comunicación social (1986-1991). El autor describe el planteamiento teórico que le permite abordar el análisis de la mediación comunicativa que, por una parte, construye 
como objeto de conocimiento la noción de catástrofe a partir de su expresión/ representación y, por otra, estudia la mediación que se ejerce a través de la práctica social de la divulgación científica en los medios. Otro trabajo que podríamos incluir entre los estudios sobre divulgación científica, es la tesis que defendió, en 1996, Wilma Peregrino de Morais, en la Universidad Autónoma de Barcelona, titulada El periodismo y el arte de contar historias. Un estudio acerca de la construcción de la noticia científica. En este trabajo la autora analiza cómo la prensa diaria divulga la ciencia en función de tres momentos distintos que desarrolla a lo largo de la investigación: a) el momento en que se decide en función del público al que va dirigido qué debe ser difundido; b) cómo construir los acontecimientos científicos que han sido seleccionados para ser difundidos; y c) hasta qué punto el acontecimiento ha de ser destacado. También en 1996, Bienvenido León Anguiano presenta la tesis El documental de divulgación científica. Estudio de las técnicas empleadas por David Attenborough, en la Universidad de Navarra. Uno de los objetivos de este trabajo es identificar las claves de la eficacia comunicativa del documental de divulgación científica. Como caso de estudio, se centra en el análisis de las obras del guionista y presentador británico David Attenborough. En 1998, María José Baselga Fuster defiende la tesis doctoral La infografía médica y sanitaria en el proceso de divulgación científica en España, en la Universidad Complutense de Madrid. Como núcleo central de la investigación, analiza formal y cualitativamente los suplementos de salud de la prensa donde encuentra una abundante muestra de infográficos, que ayudan al lector a identificar los contenidos de la información con rapidez y claridad. En 1999, Mariano Belenguer Jané defiende la tesis Periodismo de viajes: análisis de una prensa especializada, en la Universidad de Sevilla. El autor incluye antecedentes históricos de los textos periodísticos de viajes en los que vincula las relaciones que existen entre el periodismo de viaje y la divulgación científica.

\section{c) Investigaciones sobre prensa científica desde Periodismo}

También hemos de destacar aquellas investigaciones sobre prensa especializada abordadas desde los departamentos de Periodismo, aunque esta línea de trabajo, desde las facultades de Ciencias de la Información ha sido tratada excepcionalmente. Cabe citar, como ejemplo, el estudio de Juan José Fernández Sanz sobre la prensa veterinaria hasta 1903. Fernández Sanz defiende en 1993, en la Universidad Complutense de Madrid, la tesis doctoral titulada La prensa médicofarmacéutica y veterinaria (1883-1903). Este autor aborda el estudio de la prensa médico-farmacéutica y veterinaria entre 1883-1903, enlazándolo con el libro de Méndez Álvaro ${ }^{8}$ y concluyendo en el año 1903, momento en el que se celebró en

8 Cfr. MÉNDEZ ÁLVARO, Francisco: Historia del periodismo médico y farmacéutico en España. Universidad de Valladolid, Valladolid, 1978. 
Madrid el segundo congreso internacional de prensa médica. En este trabajo, el autor -que aborda la investigación desde el punto de vista histórico-periodísticocatalogó 515 revistas del gremio, considerándose, pues, la prensa científico-profesional de mayor envergadura de la época. A esta situación contribuía el desarrollo de las especializaciones médicas, la creación de los colegios oficiales de médicos y farmacéuticos, en la última década del siglo XIX, así como un persistente clima de emulación entre las plumas más relevantes del periodismo médico que buscaban órganos diferenciados de expresión?.

\subsection{La investigación en comunicación científica desde Historia de la Ciencia}

\section{a) Investigaciones sobre el tratamiento informativo de la ciencia}

A pesar del desconocimiento de la comunidad científica sobre el mundo de los medios de comunicación, proliferan -en el examen bibliográfico abordado para este artículo- los estudios sobre el tratamiento periodístico de temas científicos en la prensa diaria, realizados desde los departamentos de Historia de la Ciencia e Historia de la Medicina y, fundamentalmente, desde las facultades de Medicina y Farmacia. Con la particularidad añadida de que el análisis en la prensa de temas científicos fue objeto de estudio, en primer lugar en las facultades de Ciencias Experimentales que en las facultades de la Ciencias de la Información. Como ejemplo, localizamos la tesis doctoral titulada La información psiquiátrica en la prensa diaria, de Emilio Gamo Medina, defendida en 1988 en la Facultad de Medicina de la Universidad Complutense de Madrid. Esta tesis doctoral abre una línea de investigación en las facultades de ciencias sobre la construcción periodística de temas vinculados con la medicina y con la salud. En esta misma línea va dirigida la tesis Medicina y prensa. Análisis de la imagen de la sanidad de Joaquín López Álvarez, defendida en 1992, en la Universidad de Alcalá de Henares.

También en 1992, José Antonio Vázquez Díaz presenta en la Facultad de Medicina de la Universidad de Sevilla la tesis Análisis de diversos factores que influyen en la utilización de medicamentos: publicidad, visita médica, prensa escrita. Uno de los capítulos de esta investigación se basa en el análisis de la

9 Vid. otros trabajos de este autor sobre el espectacular desarrollo de la prensa médico-farmacéutica en las últimas décadas del siglo XIX, convirtiéndose en el sector de la prensa especializada que más pagaba por «derecho de timbre». FERNÁNDEZ SANZ, Juan José: "La Asociación de la prensa Médico-Farmacéutica (1875), pionera del asociacionismo periodístico español", en Asclepio. Revista de Historia de la Medicina y de la Ciencia, $\mathrm{n}^{\circ}$ 44, 1992, pp. 29-52; "El II Congreso Internacional de Prensa Médica (1903)", en Anuario del Departamento de Historia, no 5, 1993, pp. 185-199. Además de dos obras publicadas a raíz de la investigación llevada a cabo en la tesis doctoral: FERNÁNDEZ SANZ, Juan José: La prensa veterinaria I (hasta 1903). Aache Ediciones, Guadalajara, 1995; y La prensa homeopática española en el siglos XIX. Instituto Homeopático y Hospital de San José, Madrid, 1999. 
información publicada en la prensa diaria sobre fármacos. Para el análisis de los textos, el autor elige el diario $A B C$ de Sevilla, correspondiente al año 1991. Descubre que la patología a la que más se recurre en los textos publicados es todo lo que se vincula a los procesos neoplásicos; y las vacunas, los medicamentos más citados; la valoración global de los artículos publicados es de aceptable/excelente, en un $60 \%$ de los textos analizados, según constata el investigador.

En 1993, en la Facultad de Medicina de la Universidad Autónoma de Barcelona, Antonio Salgado Remigio defiende la tesis Descripción y valoración de la información de un suplemento semanal de medicina y salud en un periódico de Barcelona. Este trabajo trata sobre la información médica publicada en el suplemento de Medicina de La Vanguardia. El autor, además de realizar el análisis de contenido de la información médica publicada, encuestó a lectores y médicos de la provincia de Barcelona para cotejar los datos obtenidos con el estudio descriptivo de los documentos analizados, con el propósito de describir y valorar la información y la respuesta social del Suplemento Médico. Algunas de las conclusiones a las que llega Salgado en este trabajo están relacionadas con el ejercicio de la profesión periodística especializada; ya que los médicos encuestados consideraron que la labor divulgativa y de educación sanitaria que se estaba llevando a cabo desde el suplemento médico analizado era muy positiva.

En la Facultad de Farmacia de la Universidad Complutense de Madrid, Marta Castells Cuixat lee la tesis Nutrición y prensa, en 1995. Para esta investigación, la autora selecciona mensajes publicitarios y periodísticos publicados en la prensa sobre nutrición. Analiza 164 periódicos y 66 revistas (48 dirigidas a público femenino y 18 a público masculino) durante la primera semana de los seis primeros meses de 1992. En total analiza 1681 mensajes (800 publicitarios y 881 periodísticos). Con esta muestra estudia la temática, el contenido, la extensión, el tipo de mensaje, los alimentos y las dietas referidas, así como los términos y los errores habitualmente cometidos. Las conclusiones de la investigación fueron que: a) los mensajes nutricionales contienen frecuentes errores, que no contribuyen a mejorar la educación nutricional de la población; b) el control de la información nutricional contribuiría a evitar equívocos y distorsiones en un campo con grandes repercusiones en la salud de la población.

\section{b) Investigaciones sobre la prensa científica desde Historia de la Ciencia}

Por otra parte, encontramos una línea de investigación iniciada en las Facultades de Medicina, principalmente desde los departamentos de Historia de la Ciencia y desde Historia de la Medicina. Localizamos una serie de trabajos que tratan de estudiar, analizar, describir y catalogar numerosas publicaciones científicas. Desde las Facultades de Medicina, la producción de trabajos vinculados a la prensa médica es muy extensa. Son trabajos en los que se investiga sobre el origen 
y la evolución hasta nuestros días del periodismo médico a través de las publicaciones más importantes de cada una de las ramas de la Medicina; con análisis de contenido sistemático y con una estructuración racional de toda la información analizada. Y, por otra parte, encontramos cómo se analizan temas concretos a través de estas publicaciones especializadas en contenidos científicos y técnicos.

En esta línea se halla el estudio de Baltasar Llopis Mínguez titulado Las publicaciones sobre venereología en la España isabelina (1834-1868) y su posición internacional. El autor presenta esta tesis, incluida en una de las líneas centrales de investigación de la cátedra de Historia de la Medicina, en 1989, en la Facultad de Medicina de la Universidad de Valencia. En las conclusiones el autor cataloga 6.198 trabajos desde 1834 a 1868 sobre venereología y analiza detalladamente los artículos publicados sobre esta materia en el Boletín de Medicina, Cirugía y Farmacia, en la Gaceta Médica y El Siglo Médico.

Un estudio que aporta datos sobre los modelos de producción y sobre el consumo de la literatura científica médica aragonesa es la tesis doctoral que defiende Mar Mimbela Sánchez, en 1990, en la Facultad de Medicina de la Universidad de Zaragoza. La investigación se titula 'Medicina Práctica': El periodismo médico en Aragón y se centra en dos aspectos: a) el estudio de la producción científica de los autores españoles en un órgano de expresión aragonés; y b) el estudio de la comunicación científica que la comunidad aragonesa mantiene con la comunidad científica nacional e internacional.

En 1993, en la Facultad de Medicina de la Universidad de Cádiz, José Eduardo Lasarte Calderay presenta La prensa de las profesiones auxiliares sanitarias en Andalucía. Estudio socio-profesional (1916-1939). En esta investigación se realiza por primera vez una valoración de conjunto de la prensa profesional producida por los auxiliares sanitarios en Andalucía en el siglo XX. El autor ha localizado un total de 444 números de ocho revistas andaluzas generadas por los auxiliares sanitarios entre 1916 y 1939, que se desglosan de la siguiente forma: $E l$ practicante gaditano, 184; El auxiliar médico de Córdoba, 17; La matrona hispalense, 4; Federación sanitaria de Sevilla, 4; Boletín de los practicantes de Granada, 1 y El practicante malagueño, 1 . En estas revistas se informa sobre reivindicaciones laborales, organización colectiva, movimientos asamblearios de estos profesionales y sobre el intrusismo profesional, como uno de los problemas más debatido y denunciado por los practicantes.

En el año 1993, también en la Facultad de Medicina de la Universidad de Cádiz María del Carmen Sebastianes Marfil presenta la tesis La anestesia general en Andalucía a través de su prensa médica (1847-1936). El trabajo se hace en función de la escasez de estudios sobre la materia anestesiológica en la prensa médica de Andalucía. El material empleado corresponde a 26 colecciones periódicas editadas en las provincias andaluzas desde 1847 hasta 1936, ambas inclusive, de las que se han extraído 162 artículos sobre la anestesia general. 
En este mismo año, en la Facultad de Medicina de la Universidad de Barcelona, Teresa Casanovas Taltavull presenta la tesis La aportación de la 'Gaceta Médica Catalana' a la configuración y desarrollo de la patología digestiva en Cataluña (1878-1921). El material se basa en los artículos publicados en la Gaceta Médica Catalana sobre patología digestiva. La Gaceta fue una revista quincenal, dirigida al médico generalista, que se publicó en Barcelona desde 1878 hasta 1921.

Carlos Pinto Madroñero defiende en la Facultad de Medicina de la Universidad de Alicante, en 1995, El periodismo médico español contemporáneo a través de la 'Revista Clínica Española' (1940-1968). Pinto Madroñero utiliza, como fuente para su análisis, la Revista Clínica Española (1940-1968) hasta el fallecimiento de su fundador, Carlos Jiménez Díaz. En la primera parte de la tesis doctoral, el autor realiza un análisis estadístico descriptivo y bibliométrico de los artículos originales de la publicación, así como un estudio de las secciones de la revista y su ubicación en el contexto histórico-científico español. En la segunda parte, lleva a cabo un análisis interno del conjunto de artículos relativos a la cirugía cardiaca, cuya presencia constituye un punto de partida muy significativo sobre la historia de esta rama especializada de la cirugía española del período objeto de estudio.

En 1995, Gabriel Oliver Capo defiende Análisis histórico de la 'Revista Balear de Ciencias Médicas (1885-1912), en la Facultad de Medicina de la Universidad de Zaragoza. El trabajo presentado consiste en el análisis de una revista que constituye el esfuerzo médico científico más importante de la Mallorca decimonónica y de principios del siglo XX. Este autor analiza el contenido de la revista, pero ante la imposibilidad de realizar un análisis exhaustivo selecciona, a modo de muestra, los artículos originales publicados sobre otorrinolaringología.

Un año más tarde, en 1996, Raquel Sáez Villaverde defiende la tesis Los comienzos del periodismo médico español: Boletín de Medicina, Cirugía y Farmacia. Tercera serie 1846-1850, en la Facultad de Medicina de la Universidad de Valladolid. Esta investigación trata sobre los inicios del periodismo médico del siglo XIX llevada a cabo a través de un análisis sistemático y de una estructuración racional de la totalidad de la información contenida en la revista médica Boletín de Medicina, Cirugía y Farmacia, una de las revistas profesionales más importantes de la pasada centuria. Se destaca el carácter profesional de la publicación y de enlace entre los descubrimientos científicos médicos de la época y la clase médica a través de los 2.452 artículos que componen esta serie.

Todos estos trabajos contribuyen a incrementar la producción científica española en una determinada rama de la ciencia, y más concretamente de la Medicina, vinculando el carácter profesional de la publicación estudiada con los descubrimientos científicos y médicos de la época durante la que se edita. 


\section{Contexto en el que evoluciona el concepto de periodismo científico. De los Mercurios a los diarios digitales}

¿El periodismo científico evoluciona como especialización en el marco del periodismo informativo durante el siglo XX o se puede ya considerar el inicio de la especialización y de la divulgación en la prensa en el contenido publicado en los mercurios o en las gacetas? Calvo Hernando señala que el nacimiento del periodismo científico se podría situar en el origen del periodismo en general. Sin embargo, este autor marca el nacimiento del periodismo científico en la Revolución Industrial y en la necesidad de difundir la ciencia a los profesionales que estaban vinculados con la maquinaria destinada a la producción industrial ${ }^{10}$.

El profesor Casasús destaca en un trabajo sobre los orígenes y la evolución del periodismo científico en Cataluña que el alemán Christian Weise publicó en 1676 uno de los primeros análisis de contenido de prensa, examinando las noticias de los nuevos periódicos alemanes entre 1660 y 1676 . Y, en este marco histórico de finales del siglo XVII, el médico y teólogo, Tobias Peucer, es quien presenta la primera tesis doctoral -que se conoce en el mundo- sobre periodismo. El trabajo titulado De relationibus novellis fue defendido en la Universidad de Leipzig en 1690. En la tesis de este autor, según Casasús, se constata la aparición de dos cuestiones vinculadas al periodismo científico y de precisión: “...a) la confiança teòrica que els "nous periòdics" -així anomenava aquell doctorand els diarismillorarien la narrativa històrica i la mateixa ciència de la història (Peucer, 1990, 38-39); i b) els criteris sobre quins esdeveniments caldria seleccionar per als "nous periòdics", entre els quals es esdeveniments caldrà que prevalgui "un valor memorable o científico" (Peucer, 1990, 39-40)..."11

Es evidente que antes de la Revolución Industrial ya existían gacetas y periódicos que incluían entre sus contenidos temas de carácter científico, aunque todavía no estaban tratados como textos periodísticos porque no se regían por los principios de actualidad y de objetividad informativa; dos elementos fundamentales que definen el quehacer de la profesión periodística y sus distintas especializaciones.

Según Bosch Carrera, en el siglo XVIII la prensa fue una ventana a la Ilustración, ya que presidió todos los esfuerzos por divulgar las novedades técnicas y los principios científicos. En la época del Diario de los Literatos de España, nacido en abril de 1737, el 27\% de los periódicos publicados presentaban, según esta misma autora, un claro interés por los temas de divulgación científica y

10 Cfr. CALVO HeRnANDo, Manuel: Op. cit.. 1992, p. 9; y Manual de Periodismo Científico. Bosch, Barcelona, 1997, p. 22.

11 CASASÚS i GURI, Josep Maria: "Els orígens i l' evolució del periodisme científic a Catalunya", en Gazeta, no 1, 1994, p. 109. También, vid. PEUCER, Tobias: "Sobre els relats periodístics", en Periodística, no 3, 1990, p. 38-40. 
técnica. Con este periódico llegó a nuestro país la moda europea de los periódicos «sabios» o «eruditos», aunque tardíamente.

En la época de El Pensador (1762), como en la de El Censor (1781), se produjo un cambio, un descenso en la publicación de temas tecnocientíficos. Tan sólo el 16\% de los periódicos de los años sesenta del siglo XVIII se ocupaban de estos temas; aunque por entonces hay que destacar siempre la labor de F. Mariano Nipho, periodista prolífico que abarcó todos los campos del periodismo ${ }^{12}$. En los años ochenta, el porcentaje de la publicación de temas científicos en la prensa se reduce algo más, aunque la cifra no es significativa cuantitativamente, en relación con las dos décadas anteriores, esto es, un $15,3 \%$ del total de los periódicos ${ }^{13}$.

Según Guinard, durante el siglo XVIII, la prensa española contaba con publicaciones de temáticas muy variadas: “(...) publicaciones consagradas a las cuestiones científicas, técnicas y económicas, periódicos de divulgación (...) revistas literarias y semanarios consagrados principalmente a la crítica de la sociedad y las costumbres, entre los cuales se encuentran los 'espectadores' (...) También existe una prensa balbuciente en provincias en la que es más difícil llevar a cabo una clasificación"14.

En esta misma línea, la profesora Sáiz señala que a partir de 1750 destacan fundamentalmente, dentro de las publicaciones sobre ciencia y divulgación, aquellas que tenían un carácter económico. En su obra, esta autora destaca el hecho de que los periódicos comenzaran a tener un subtítulo como elemento de orientación sobre los contenidos que trataban. Como ejemplo cita los Discursos mercuriales de J. Enrique De Graef, con varios números publicados entre 1752 y 1756. Sáiz estima que entre 1755 y 1756 aparecieron veinte números y que De Graef subtituló su periódico como «Memorias sobre Agricultura, Marina, Comercio y Artes Liberales y Mecánicas», dedicando numerosos artículos a desarrollar todas estas materias $^{15}$.

En relación con la evolución y el desarrollo a lo largo de la historia de la información científica y técnica, los profesores Esteve y Fernández del Moral señalan que los inicios de la divulgación científica están unidos al nacimiento y al desarrollo de las Academias de las Ciencias que fueron surgiendo en el siglo

12 En 1758, Nipho puso en marcha la primera publicación diaria española: Diario noticioso, curioso, erudito y comercial, público y económico. Esta publicación fue precedida únicamente en Europa por tres diarios británicos. Cfr. PIZARROSO QUINTERO, Alejandro: De la Gazeta Nueva a Canal Plus. Editorial Complutense, Madrid, 1992, p. 35.

13 Cfr. BOSCH CARRERA, María Dolors: "Los inicios de las revistas especializadas en España", en Hispania, $\mathrm{n}^{\circ}$ 52, 1992, pp. 263-277; y: "Costumbres y opiniones en la prensa española del siglo XVIII", en Pedralbes. Revista d'Historia Moderna, n 9, 1989, pp. 171-180.

14 Citado en SÁIZ, María Dolores: Historia del periodismo en España. Los orígenes. El siglo XVIII. Alianza Madrid, 1990, p. 120.

15 SÁIZ, María Dolores: Op. Cit., 1990, p. 121. 
XVIII: "En España, una de las primeras publicaciones científicas es el periódico Efemérides barométrico-médicas matritenses, en 1734, boletín de la recientemente constituida Academia de Cirugía y Medicina, compuesto fundamentalmente de traducciones de publicaciones médicas francesas. En 1736 surge Memorias eruditas para la crítica de arte y ciencia. En 1737 se publica en Madrid el Diario de los literatos de España y en 1833 se edita en Barcelona El Vapor, diario científico, literario, económico, agrónomo y mercantil" ${ }^{16}$.

Durante la mayor parte del siglo XIX, la profesora Seoane señala que el periódico era «fundamentalmente un arma de combate político», ya que la prensa se consideraba la expresión de las distintas facciones políticas o de los grupos de interés que se imponían en cada momento ${ }^{17}$.

Un ejemplo de periódico científico que destaca esta autora es la Crónica Científica y Literaria, de José Joaquín Mora, que nace en 1819 y que una vez que entra en el Trienio Liberal cambia su orientación científica en política. En referencia a los temas que trataba este periódico, antes del cambio político, Seoane describe en su obra lo siguiente: "Como en toda época de rígido control, la prensa se refugia en los temas científicos, técnicos y literarios. En la Crónica de Mora encontramos artículos sobre las aplicaciones del vapor a la industria, las primicias del uso de la litografía en Francia, sobre las operaciones de cataratas llevadas a cabo en Suiza, sobre los globos aerostáticos...".

Tras una larga búsqueda bibliográfica, no hemos encontrado para el siglo XIX ningún trabajo de investigación en el que se haya analizado el contenido de los temas científicos de los incipientes diarios de «referencia». Por lo tanto, sería una aporía propiciar cualquier inferencia sobre el porcentaje de información científica publicada en la prensa diaria.

Sin embargo, la conformación de la moderna prensa informativa desde mediados del siglo XIX y principios del XX, debería marcar el inicio de nuevos trabajos en el ámbito de la especialización científica en los periódicos diarios, ya que es el momento en el que se configura una prensa informativa, de actualidad y objetiva. Un ejemplo ilustrativo de esto que decimos -que ha sido constatado entre otros autores por la profesora Seoane, al establecer una relación entre las épocas de control y de censura con el desarrollo de los contenidos científicos en la prensa- se manifiesta con la aparición en los diarios españoles, en plena dictadura de Primo de Rivera, de secciones específicas de temática científica. Estudios realizados en los ámbitos locales y regionales avalan esta afirmación.

El nacimiento de secciones regulares de contenido científico es una realidad en nuestro país en los años veinte, al socaire de la difuminación de los contenidos

16 ESTEVE RAMÍREZ, Francisco y FERNÁNDEZ DEL MORAL, Javier: Áreas de especialización periodística. Fragua, Madrid, 1999, p. 110.

17 SEOANE, María Cruz: Historia del periodismo en España. El siglo XIX. Alianza, Madrid, 1989, p. 15. 
políticos y, de otra naturaleza, críticos con el régimen vigente. Pero, al mismo tiempo, es resultado de la modernización empresarial e informativa del periodismo español de la época y de una sociedad que empieza a ser permeable a los avances cientifico-técnicos del momento. En este mismo sentido, parece clara, igualmente, la existencia de una incipiente demanda social de dichos contenidos.

El estudio del profesor García Galindo sobre la prensa malagueña del primer tercio del siglo XX permite corroborar nuestras afirmaciones anteriores. Según los datos que aporta este autor, a comienzos de los años veinte el periodismo semanal malagueño cuenta con secciones de divulgación científica (La Unión Ilustrada, a partir de 1922); y a mediados de la misma década será el periodismo diario el que consolide este tipo de oferta informativa, con secciones fijas de temática científica (La Unión Mercantil, a partir de 1925) ${ }^{18}$.

A principios del siglo $\mathrm{XX}$, la prensa vive numerosas transformaciones tecnológicas que van a repercutir en la mejora de la calidad del periódico como bien de consumo. El profesor Gómez Mompart sistematiza los cambios que caracterizaron el origen de la prensa de masas en las siguientes claves: a) estructuras narrativas, b) progreso técnico, c) redacción y d) contenidos-signos de modernidad (se tematizan muchas informaciones de la prensa, en política, en consumo, en entretenimientos públicos, en páginas especiales y suplementos) ${ }^{19}$.

La evolución producida en el periodismo en estos cuatro frentes en las primeras décadas del siglo XX conduce al desarrollo de los diarios de masas. Destacamos, pues, en el proceso evolutivo de la prensa diaria a lo largo de esta centuria, un hecho crucial en la producción de la información como es la segmentación de los contenidos, la tematización de las agendas y, por tanto, la división de las redacciones en áreas de especialización ${ }^{20}$.

Tal y como hemos comprobado hasta el momento, la evolución experimentada por el periodismo científico durante el primer tercio del siglo XX, gracias a la atención prestada por los grandes diarios españoles de la época, sufrirá un serio revés con la Guerra Civil y un vacío informativo temático tras la guerra, pues la prensa del Movimiento no favoreció la aparición de secciones científicas estables.

18 GARCÍA GALINDO, Juan Antonio: La prensa malagueña, 1900-1931. Estudio analítico y descriptivo. Ayuntamiento de Málaga, Málaga, 1999, pp. 256 y 262-263.

19 GÓMEZ MOMPART, Josep Lluís: “¿Existió en España prensa de masas? La prensa en torno a 1900”, en ÁlVAREZ, Jesús Timoteo et al.: Historia de los medios de comunicación en España. Periodismo, imagen y publicidad (1900-1990). Ariel Comunicación, Madrid, 1989, pp. 27-40.

20 Evidentemente el periodismo científico no se ejerce en un diario desde una sección de Ciencia y Tecnología. De hecho, hasta la década de los años 80 y de los años 90 del siglo XX no se comienzan a incluir páginas o secciones especializadas en Ciencia y Tecnología o Suplementos Especializados en los periódicos de referencia de todo el estado español. Pero lo que sí han analizado los expertos en periodismo especializado es que se producen divisiones temáticas en las redacciones en: Internacional, Economía, Política, Sociedad, Deportes, y Cultura y Espectáculos. Los periodistas científicos trabajaban desde la sección de Sociedad, en la que también se incluyen otros temas como Religión, Sucesos, Educación y Medicina, etc. 
Según afirma Gómez Mompart, la única tematización que existía en la prensa en las dos primeras décadas tras la guerra era la exaltación del régimen: "El periodismo que mayoritariamente se practicó en la prensa, durante las dos primeras décadas de la posguerra, tenía un estilo insulso y mediocre, con una presentación gris y sin apenas ilustraciones y con una compaginación desordenada, con casi nulo fotoperiodismo, destacando una tematización que era la exaltación del régimen, cargado de excesivas servidumbres y con un discurso periodístico de ínfima calidad, lenguaje doctrinario y oficialista, léxico autoritario y moralino y con unos mensajes redundantes donde el ejército, la iglesia y la unidad de la patria eran las letanías ${ }^{21 "}$.

El oscurantismo del discurso en la prensa durante el franquismo, en general, y sobre ciencia y tecnología, en particular, empieza a vislumbrar algo de luz a finales de los sesenta, sobre todo, por el impacto social que producen los temas tratados por la televisión, como la llegada del hombre a la Luna, o el primer trasplante de corazón. Sin llegar a ser la ciencia ni la tecnología temas tratados por los periódicos desde una perspectiva filosófica-crítica sino de forma espectacularizante o llamativa, los eventos científicos de gran magnitud (sobre todo, con la llegada de la producción audiovisual) son destacados en las agendas informativas con las prerrogativas propias del régimen.

En la actualidad, el conocimiento de los hechos científicos a través de la prensa de información general es minoritario dado el escaso índice de lectores de periódicos, frente a medios de comunicación masivos, como la televisión. Para que se haya propiciado esta comunicación masiva del conocimiento científico hemos tenido que llegar a la segunda mitad del siglo XX, y que los ciudadanos hayan podido acceder a la actualidad científica a través de los medios audiovisuales. Desde esta perspectiva, el profesor Pierre Fayard ha acuñado el término Comunicación Científica Pública, subrayando, de este modo, el carácter público de la información científica que se publica y se emite mediante técnicas de difusión masiva $^{22}$.

Sin embargo, parece conveniente señalar que éste ha sido un proceso gradual, que, al menos en el caso español, ha tenido que ver con nuestra historia contemporánea. Uno de los episodios más significativos que, a modo de ejemplo, puede ilustrar lo que decimos, tiene que ver con la respuesta de los intelectuales y científicos españoles ante la crisis del 1898. A comienzos del siglo XX cientí-

21 GÓMEZ MOMPART, Josep Lluís: "Ecosistema comunicativo franquista y construcción simbólica y mental de España”, en La comunicación social durante el Franquismo. Servicio de Publicaciones de la Diputación Provincial de Málaga, Málaga, 2002 (en prensa).

22 En 1972, John Ziman ya había destacado que la ciencia era «conocimiento público», ya que este autor consideraba que el carácter de publicidad del conocimiento era lo esencial para su verificabilidad. Cfr. FAYARD, Pierre: La communication scientifique publique. Chronique Sociale, Lyon, 1988; y ZIMAN, John: El conocimiento público. Fondo de Cultura Económica, México, 1972. 
ficos e intelectuales cifraban la regeneración de España en el progreso científico y en la revitalización de la Universidad. Inquietud que tenía sus antecedentes inmediatos en el krausismo y en la Institución Libre de Enseñanza.

El profesor Mainer, recoge, a este respecto, diversos testimonios publicados en El Liberal de Madrid. Por ejemplo, para Santiago Ramón y Cajal, uno de los remedios de la crisis era "crear ciencia original en todos los órdenes del pensamiento: filosofía, matemáticas, química, física, biología, sociología, etc. Tras la ciencia original -decía el insigne científico- vendrá la aplicación industrial de los principios científicos, pues siempre brota al lado del hecho nuevo la explotación del mismo, es decir, la aplicación al aumento y a la comodidad de la vida" ${ }^{23}$.

Sorprende, a los propósitos de este artículo, la presencia del periodismo de la época en este discurso regenerador - en el que la ciencia tiene cabida principal -. Las palabras de Ramón y Cajal formaban parte de una encuesta que el diario madrileño El Liberal llevó a cabo en los primeros años del siglo XX entre hombres importantes del país. Otro de los encuestados, citado también por el profesor Mainer, fue Joaquín Costa, quien atribuía a personas y a centros concretos la posibilidad de salida a la crisis finisecular: "Educadores del Museo Pedagógico, de la Normal, sociólogos de la Universidad de Oviedo, Salamanca, colonistas de la Geográfica, hidráulicos de Aragón, financieros de las Cámaras y Círculos industriales y mercantiles, y algunos periodistas, muy pocos; si hay posible redención -decía Costa-, ellos tienen la clave" ${ }^{24}$.

Pese a la apreciación cuantitativa que hace Costa sobre los periodistas como factor de regeneración, su sola consideración -realizada además en las páginas de un medio de información-, muestra, sin lugar a dudas, el papel que estaban adquiriendo periódicos y periodistas en la regeneración del país, y por ende en la divulgación científica como herramienta de esa misma regeneración.

El profesor Fernández del Moral estima que el nacimiento del moderno periodismo científico surge a raíz del lanzamiento, en agosto de 1945, de la bomba de Hiroshima, porque considera que es cuando se hace muy perceptible la línea que existe entre los científicos y la sociedad, que sólo se puede traspasar mediante el periodismo ${ }^{25}$. La sociedad se da cuenta de que necesita saber en qué líneas de investigación se está trabajando para poder aceptar resultados y ejercer un control sobre los desarrollos científicos y tecnológicos.

Fernández del Moral destaca que el momento más crítico que ha vivido la Humanidad como consecuencia de la aplicación de un avance científico fue, sin

23 Citado en MAINER, José Carlos: Regionalismo, burguesía y cultura: Revista de Aragón (1900-1905) y Hermes (1917-1922). Guara Editorial, Zaragoza, 1982, pp. 52-53.

24 Ibídem, p. 53.

25 FERNÁNDEZ DEL MORAL, Javier: "La especialización periodística como nexo necesario entre ciencia y sociedad", en ESTEVE RAMÍREZ, Francisco (Coord.): Estudios sobre información periodística especializada. Fundación Universitaria San Pablo CEU, Valencia, 1997, p. 9. 
duda, la explosión de la primera bomba atómica sobre la ciudad japonesa de Hiroshima. Ese momento histórico fue decisivo para que toda la comunidad internacional expresara la necesidad del control científico ${ }^{26}$. Explica este autor que surge así la idea de corresponsabilidad, es decir, la idea de responsabilidad compartida entre el científico y la humanidad, entre la ciencia y la sociedad cuyo nexo es el periodismo científico ${ }^{27}$.

Para compartir esta responsabilidad es necesario que la sociedad participe en la ciencia. Por tanto, para que los individuos participen en las decisiones parlamentarias científicas y técnicas, es necesario que exista una opinión pública bien informada a través de los medios de comunicación. A este respecto, el periodista Tito Drago, miembro del Instituto Internacional de Prensa y fundador de la agencia de noticias Inter Press Service (IPS), señala que para que exista una responsabilidad compartida entre sociedad y científicos tiene que producirse un debate interdisciplinario permanente para acercar la ciencia a los ciudadanos y los ciudadanos a la ciencia a través de los medios de comunicación ${ }^{28}$.

Una de las nuevas líneas del periodismo actual es el 'periodismo de servicio' pues supone el avance de los ciudadanos hacia el consumo selectivo de la información. Esta especialización del periodismo ha configurado un panorama mediático muy interesante para la tematización en ciencia y tecnología (actualmente con un mayor incremento de las páginas dedicadas a salud y nuevas tecnologías, en prensa, y con programas de divulgación en radio y televisión).

Según la profesora Diezhandino el periodismo de servicio resuelve o, más concretamente, responde a una demanda social de cuestiones que preocupan a los ciudadanos, que versan, entre otras, sobre las medidas preventivas contra el cáncer o el SIDA o sobre la información meteorológica ${ }^{29}$. Ciertamente, en el conjunto de la información especializada en ciencia, los temas sobre biomedicina ocupan un lugar importante en las agendas informativas, por el interés que originan en los lectores. Para esta autora, el periodismo de servicio atiende al individuo más que al ciudadano, «es más personalizante que socializante», ya que interesa más la utilidad en la vida personal que en la social.

Revisando los escenarios futuribles de la comunicación científica, sin especulaciones prospectivas, encontramos una línea de investigación innovadora; esto es, el desarrollo de este periodismo especializado en los próximos años a través de los nuevos medios de comunicación. Se trata, pues, del tratamiento informativo que recibirá la ciencia y tecnología a través de Internet.

26 Ibíd., p. 8.

27 Ibíd., p. 9.

28 Vid. DRAGO, Tito (Comp.): "Una responsabilidad compartida”, en Arbor, no 534-535, 1990, p. 9-11.

29 Vid. DIEZHANDINO NIETO, María Pilar: "El periodismo de servicio, la utilidad en el discurso periodístico", en Anàlisi, no 15, 1993, pp. 117-125. 
Las redes de comunicación han abierto un nuevo canal o fuente de información que nos permitirá seguir planteando cuestiones relativas a la divulgación del conocimiento científico, a través de medios de comunicación, ya que éstas proporcionan una mayor interacción con los individuos y, por tanto, una retroalimentación para la rutina periodística.

Un buen ejemplo de la complejidad y, al mismo tiempo, de las posibilidades que para los lectores tiene la prensa digital es el hecho de que estos medios tengan abiertos debates o foros de discusión sobre temas científicos en los que se puede interactuar con otros lectores y/o con los redactores del periódico. Por lo que se plantean nuevos aspectos para la investigación: ¿Pueden llegar a participar los lectores en la producción de la noticia científica? ¿Qué grado de interés generan en los lectores los debates sobre impactos sociales de nuevos desarrollos tecnocientíficos en la prensa on-line? y ¿cuál es el índice de participación de los lectores en temas sobre ciencia y tecnología?

La verdadera revolución tecnológica de la sociedad de la información se halla en la multiplicación de las formas de comunicación, y en la posibilidad que se abre a la intercomunicación a distancia. Es lo que Lazar denomina "sociedad comunicacional", aquélla en la que cada individuo podría estar en relación con todo el mundo. El universo de los media -escribe esta autora- ya no está limitado a los mass media. El satélite, el magnetoscopio, el correo electrónico forman ya parte del universo comunicativo de los hombres ${ }^{30}$.

Así las cosas, el acceso de los lectores de los diarios digitales a la información científica y técnica, no sólo quedará restringida a la información seleccionada por los medios sino que, en función del interés personal o profesional, podrá acceder a otras fuentes de información primaria (a través de los resultados experimentales de los propios laboratorios farmacéuticos o a través de las investigaciones abordadas en los centros de investigación públicos y privados).

Este nuevo universo comunicativo -en el que la tecnología va muy por delante de los contenidos- sigue siendo, sin embargo, desigual. Pues la disponibilidad y el acceso a los nuevos medios vienen determinados por factores tecnológicos y económicos. Los desequilibrios económicos están configurando igualmente un panorama mediático desigual.

Los beneficios que indudablemente comportan estas nuevas tecnologías de la comunicación se producirán en primer lugar, como así ocurre, en los países de mayor desarrollo económico, y su expansión vendrá determinada por los criterios de quienes detentan la tecnología. La colonización tecnológica se produce, pero también puede producirse el aislamiento tecnológico.

30 LAZAR, J.: Sociologie de la communication de masse. Armand Colin, París, 1991, pp. 199-200. 
Actualmente, el progreso tecnológico permite la comunicación instantánea de la palabra y de la imagen animada, provocando reacciones simultáneas de la opinión pública frente a los acontecimientos. Todo esto rompe con la relación tradicional de los ciudadanos con los hechos, y a su vez con los mismos medios de comunicación. Junto a esta capacidad de los medios para informar al individuo sobre una realidad científica sin fronteras, se abre también la posibilidad de penetrar en solitario en ese universo a través de las redes, sin mediadores.

(Recibido el 15-5-2002, aceptado el 20-6-2002) 